\title{
Osteogenic potential of embryonic stem cells in tooth sockets
}

\author{
HYUN KI KANG ${ }^{1}$, SANGHO ROH ${ }^{1}$, GABSANG LEE ${ }^{1}$, SEONG-DOO HONG ${ }^{2}$, \\ HOIN KANG ${ }^{1}$ and BYUNG-MOO MIN ${ }^{1}$
}

\begin{abstract}
${ }^{1}$ Department of Oral Biochemistry and Craniomaxillofacial Reconstructive Science, Dental Research Institute and BK21 CLS,
${ }^{2}$ Department of Oral Pathology, Seoul National University School of Dentistry, Seoul 110-749, Korea
\end{abstract}

Received January 16, 2008; Accepted February 26, 2008

\begin{abstract}
Embryonic stem cells (ESCs) are established from blastocysts and give rise to various types of cells and tissues. In the present study, we assessed the osteogenic potential of ESCs using in vitro culture conditions and in vivo differentiation in tooth sockets. An ESC-derived embryoid body (EB) was formed and subsequently induced to an osteogenic lineage. The differentiated EB cells exhibited increased expression of various osteogenic markers as determined by real-time PCR analysis. Likewise, the differentiated EB-derived cells had enhanced alkaline phosphatase activity and calcium accumulation, as determined by cytochemical methods. For in vivo transplantation, mixtures of ESCs and hydroxyapatite/ tricalcium phosphate particles or EBs alone were transplanted into female rat tooth sockets. After 12 weeks, we observed formation of osteogenic structure in the tooth sockets without evidence of teratomas. These data suggest that pluripotent ESCs can serve as an alternative source for the reconstruction of craniofacial structures, as well as for further applications.
\end{abstract}

\section{Introduction}

Embryonic stem cells (ESCs) are pluripotent cells derived from the inner cell mass of a preimplantation blastocyst and are capable of differentiating into various cell types (1). Such ESCs provide an in vitro model system for studying controlled differentiation of ESCs into specific lineages, which can be useful for tissue engineering and other biomedical applications (2). For craniofacial reconstruction, a reliable source of bone or osteogenic tissue is an essential factor (3). To this end, the regeneration of osteogenic structures has been studied with stem/progeintor cells from various sources,

Correspondence to: Dr Byung-Moo Min, Department of Oral Biochemistry and Craniomaxillofacial Reconstructive Science, Seoul National University School of Dentistry, 28 Yeonkun-Dong, Chongno-Ku, Seoul 110-749, Korea

E-mail: bmmin@snu.ac.kr

Key words: embryonic stem cell, differentiation, embryoid body, transplantation, tooth socket, osteoid including exfoliated human deciduous teeth, postnatal dental pulp, and periodontal ligaments (4-7).

For regeneration of osteogenic structures, several lines of evidence need to be considered, namely, limitations of in vitro expansion of adult derived cells, recapitulation of the embryonic developmental processes necessary for the initiation of osteogenesis, and the proper response of adult-derived cells to mechanical and/or chemical signals in the oral environment. In the present study, we demonstrated successful in vitro differentiation of mouse ESCs toward osteogenic lineages under different culture conditions, and we showed that mouse ESCs mixed with hydroxyapatite/tricalcium phosphate (HA/ TCP) or ESC-derived embryoid bodies (EBs) were capable of forming an osteoid structure in a tooth socket environment.

\section{Materials and methods}

Cell culture. Mouse ESCs (J1) were cultured with mitotically inactivated feeder layers (STO cells) in a DME-based medium that consisted of DMEM (Gibco, Grand Island, NY) containing $15 \%$ fetal bovine serum (FBS; Hyclone, Logan, UT), $1 \mathrm{mM}$ sodium pyruvate, $0.1 \mathrm{mM}$ non-essential amino acids (Gibco), $4 \mathrm{mM}$ L-glutamine, $0.1 \mathrm{mM}$ ß-mercaptoethanol, $0.1 \mathrm{mg} / \mathrm{ml}$ penicillin (Gibco), $0.1 \mathrm{mg} / \mathrm{ml}$ streptomycin (Gibco), and $2.5 \mu \mathrm{g} / \mathrm{ml}$ amphotericin $\mathrm{B}$ (Gibco) at $37^{\circ} \mathrm{C}$ in a humidified $5 \% \mathrm{CO}_{2}$ atmosphere in the presence of $1,000 \mathrm{U} / \mathrm{ml}$ recombinant mouse leukemia inhibitory factor (LIF; Chemicon, Temecula, CA). ESC differentiation was induced using the hanging-drop method for 2 days. The newly formed EBs were then cultured for 3 additional days in the same medium, although it lacked LIF and contained retinoic acid $\left(10^{-7} \mathrm{M}\right.$; Sigma, St. Louis, MO) (8). MC3T3-E1 cells were maintained in a DME-based medium. For osteogenic differentiation, MC3T3-E1 cells were cultured in an $\alpha$-ME-based medium that consisted of $\alpha$-MEM (Gibco) containing 10\% FBS (Cambrex, East Rutherford, NJ), $0.1 \mathrm{mg} / \mathrm{ml}$ penicillin, $0.1 \mathrm{mg} / \mathrm{ml}$ streptomycin, and $2.5 \mu \mathrm{g} / \mathrm{ml}$ amphotericin B, supplemented with $0.1 \mu \mathrm{M}$ of dexamethasone (Sigma), $50 \mu \mathrm{g} / \mathrm{ml}$ of ascorbic acid (Sigma), and $10 \mathrm{mM} 3-$ glycerophosphate (Sigma).

In order to induce osteogenic differentiation, EBs were divided into four groups (Fig. 1b), plated onto cell culture dishes, and cultured in an $\alpha$-ME-based medium containing either $50 \mu \mathrm{g} / \mathrm{ml}$ ascorbic acid and $10 \mathrm{mM}$ B-glycerophosphate for 21 days (DAßG group); $50 \mathrm{ng} / \mathrm{ml}$ of FGF8 for 8 days and subsequently without FGF8 (eF8); $50 \mathrm{ng} / \mathrm{ml}$ of FGF8 
a

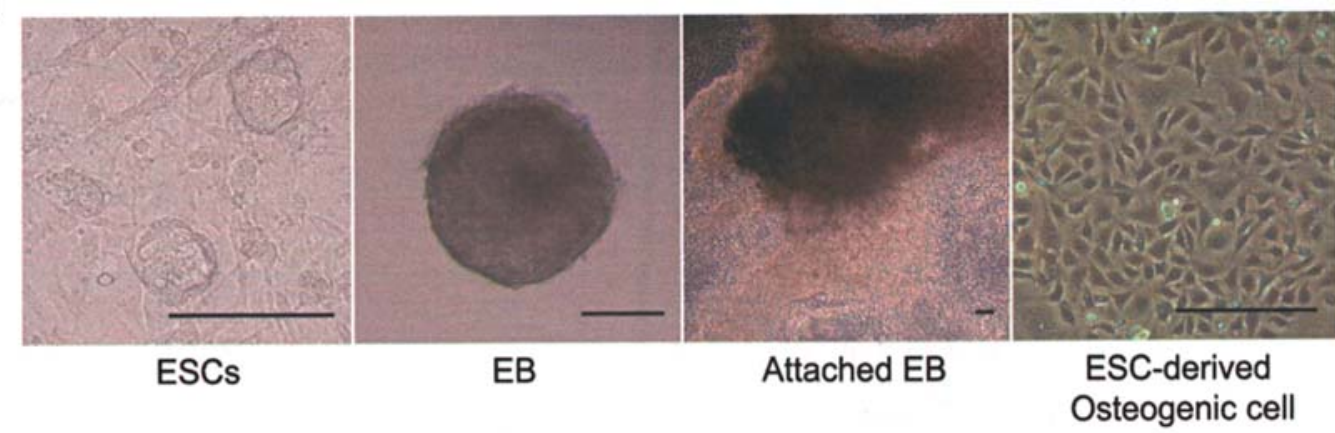

b

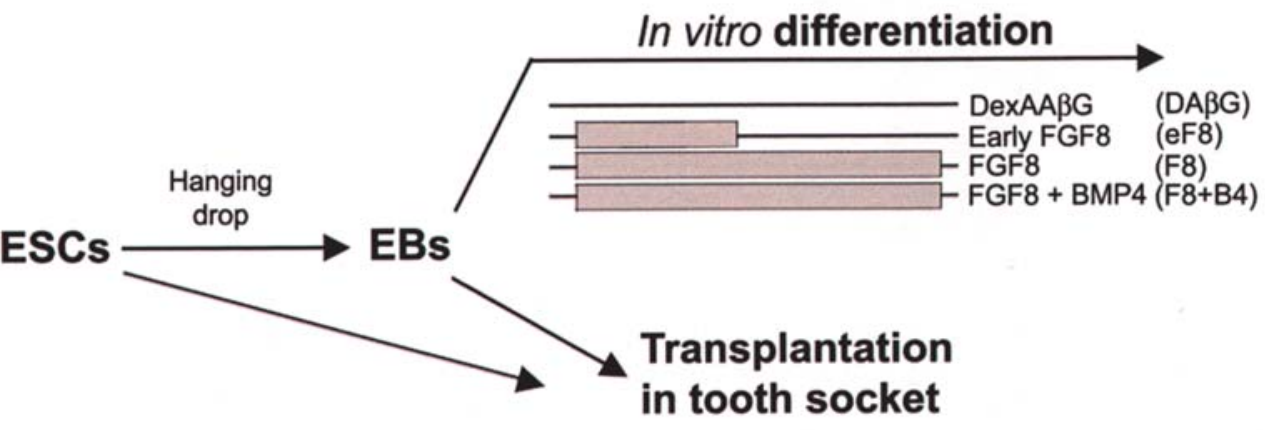

C
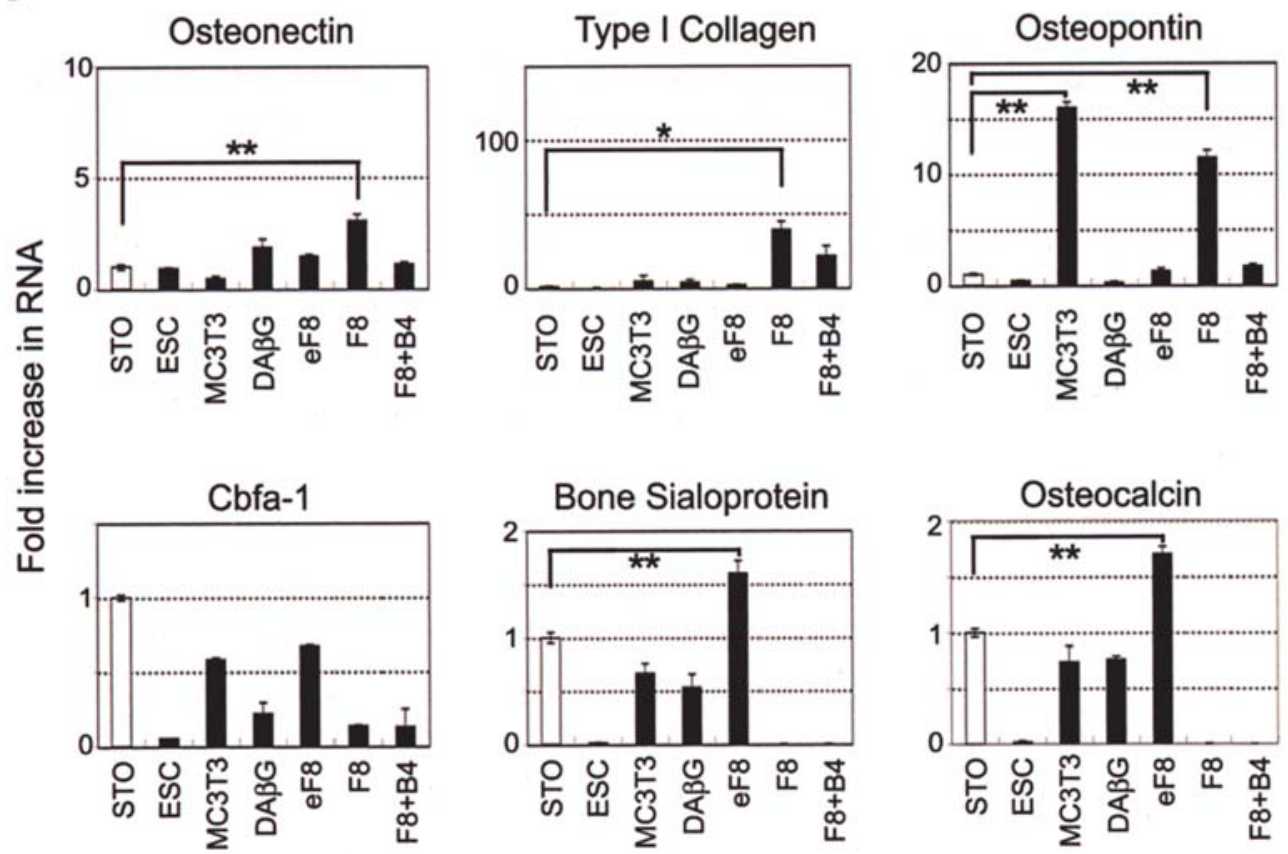

Figure 1. Osteogenic differentiation from ESCs and marker expression profile. (a) Morphology of ESCs, EB, and ESC-derived osteogenic cells. Bars, $200 \mu \mathrm{m}$. (b) Schematic image of this study. (c) Osteogenic marker expression, including osteonectin, type I collagen, osteopontin, Cbfa-1, bone sialoprotein, and osteocalcin was measured by real-time PCR analysis. ${ }^{*} \mathrm{p}<0.01 ;{ }^{* *} \mathrm{p}<0.001$.

for 21 days (F8 group); or both $50 \mathrm{ng} / \mathrm{ml}$ of FGF8 and $10 \mathrm{ng} / \mathrm{ml}$ of BMP4 for 21 days (F8+B4 group) at $37^{\circ} \mathrm{C}$ in a humidified $5 \% \mathrm{CO}_{2}$ atmosphere. After 21 days of differentiation, each group was cultured for further passages in different conditions. The DAßG group was cultured in an $\alpha$-ME-based medium; the eF8 group in a DME-based medium containing $10^{-7} \mathrm{M}$ retinoic acid; the F8 group in an $\alpha$-ME-based medium; and the $\mathrm{F} 8+\mathrm{B} 4$ group in an $\alpha-\mathrm{ME}-$ based medium.
Real-time PCR. Total RNA was isolated from the cells using the TRI-reagent (Molecular Research Center, Cincinnati, $\mathrm{OH}$ ) according to the manufacturer's instructions. After the RNA was denatured by $70^{\circ} \mathrm{C}$ incubation for $10 \mathrm{~min}$ and kept on ice for $5 \mathrm{~min}$, cDNA was prepared from $6 \mu \mathrm{g}$ of total RNA using reverse transcriptase (Invitrogen, Carlsbad, CA) and random hexamers. The resulting cDNA was subjected to real-time PCR amplification using SYBR ${ }^{\circledR}$ Green PCR Master 
Table I. Primers for major bone matrix marker genes used to assess osteoblast differentiation in real-time PCR amplification.

\begin{tabular}{lccc}
\hline $\begin{array}{l}\text { Gene } \\
\text { (NCBI ID) }\end{array}$ & \multicolumn{1}{c}{ Forward primer } & Reverse primer & $\begin{array}{r}\text { Product } \\
\text { size (bp) }\end{array}$ \\
\hline $\begin{array}{l}\text { Osteocalcin } \\
\text { (NM_001032298.2) }\end{array}$ & 5'-CCGGGAGCAGTGTGAGCTTA-3' & 5'-TAGATGCGTTTGTAGGCGGTC-3' \\
$\begin{array}{l}\text { Osteonectin } \\
\text { (NM_009242.2) }\end{array}$ & 5'-ATCCAGAGCTGTGGCACACA-3' & 5'-GGAAAGAAACGCCCGAAGA-3' \\
$\begin{array}{l}\text { Bone sialoprotein } \\
\text { (NM_001475269.1) }\end{array}$ & 5'-CAGAGGAGGCAAGCGTCACT -3' & 5'-CTGTCTGGGTGCCAACACTG-3' \\
$\begin{array}{l}\text { Osteopontin } \\
\text { (NM_009263.1) }\end{array}$ & 5'-GATGCCACAGATGAGGACCTC-3' & 5'-CTGGGCAACAGGGATGACAT-3' \\
$\begin{array}{l}\text { Collagen type I } \\
\text { (NM_007742.3) }\end{array}$ & 5'-GCATGGCCAAGAAGACATCC-3' & 5'-CCTCGGGTTTCCACGTCTC-3' \\
$\begin{array}{l}\text { ALP } \\
\text { (NM_007433.3) }\end{array}$ & 5'-GTGCCCTGACTGAGGCTGTC-3' & 5'-GGATCATCGTGTCCTGCTCAC-3' \\
$\begin{array}{l}\text { Cbfa1 } \\
\text { (NM_009820.2) }\end{array}$ & 5'-GTGCGGTGCAAACTTTCTCC-3' & 5'-AATGACTCGGTTGGTCTCGG-3' \\
$\begin{array}{l}\text { HPRT } \\
\text { (NM_013556.2) }\end{array}$ & 5'-CCTAAGATGAGCGCAAGTTGAA-3' & 5'-CCACAGGACTAGAACACCTGCTAA-3' \\
\hline
\end{tabular}

Mix (Applied Biosystems, Foster City, CA) with $300 \mathrm{nM}$ of both primers (Table I). The relative abundance of each target mRNA was calculated using the comparative cycle threshold method according to the manufacturer's instructions (Applied Biosystems).

Cytochemical analysis. Levels of alkaline phosphatase (ALP) were visualized by staining with the ES cell characterization kit (Chemicon) under the conditions recommended by the manufacturer. ALP activity was assayed in a reaction mixture composed of $8 \mathrm{mM}$ p-nitrophenyl phosphate (Calbiochem, San Diego, CA), 0.1 M glycine-NaOH buffer, pH 10.4, $150 \mathrm{mM} \mathrm{MgCl}{ }_{2}, 150 \mathrm{mM} \mathrm{ZnCl}_{2}$, and $15 \mu \mathrm{l}$ of cytosol in a final reaction volume $90 \mu 1$. The reaction was incubated for $1 \mathrm{~h}$ in a water bath at $37^{\circ} \mathrm{C}$, and was terminated by the addition of $210 \mu 1$ of $0.25 \mathrm{~N} \mathrm{NaOH}$. The absorbance was measured at $405 \mathrm{~nm}$ in a Bio-Rad Model 550 microplate reader (Bio-Rad, Hercules, CA). Enzyme activity was expressed as nmol $p$ nitrophenol product per min per mg of protein. Mineralized matrix was visualized with both von Kossa (Sigma) staining and Alizarin red staining, as previously reported (9).

In vivo transplantation into tooth sockets. The experimental animal study protocol for this study was approved by the Animal Care Committee (Seoul National University School of Dentistry), and all surgeries were performed under anaesthesia. In order to prepare the mixture of scaffold and cells (ESCs or ESC-derived EBs), porous HA/TCP (particle size of $\sim 0.8 \times 0.8 \mathrm{~mm}$; Zimmer, Warsaw, IN) was incubated with $2.2 \times 10^{6}$ cells $/ \mathrm{ml}$ ESCs for $1 \mathrm{~h}$ with an orbital shaker. Mixtures were transplanted into tooth sockets of Sprague-Dawley rats (Orient, Korea) in which both mandibular first molar teeth had been extracted. Specifically, the mixture of cells and HA/TCP was transplanted into the right tooth socket, while the HA/TCP particles alone was transplanted into the contralateral (left) tooth socket. Twelve weeks after transplantation the recipient rats were euthanized and the mandibular region of the sacrificed rats was retrieved for further study. Retrieved samples were thoroughly washed with phosphate-buffered saline (PBS; pH 7.4), fixed with $10 \%$ buffered formalin phosphate solution (Sigma) for $24 \mathrm{~h}$, decalcified with a $0.5 \mathrm{M}$ ethylenediaminetetraacetic (EDTA) solution for 6 weeks, and sectioned at a thickness of $5 \mu \mathrm{m}$. Some specimens were stained with hematoxylin and eosin (H\&E). Mason's trichrome stain was used to visualize collagen deposition.

Statistical and data analysis. The results of real-time PCR and ALP activity were compared by analysis of variance (ANOVA) using the Statistica 6.0 software package. When significant differences were found, pair-wise comparisons were performed using Scheffe's adjustment. P-values $<0.05$ were considered statistically significant.

\section{Results}

Osteogenic differentiation of mouse ESCs and marker expression profile. To induce the formation of EBs, ESCs were detached from mitotically inactivated STO feeder cells and applied to form hanging-drop cultures (Fig. 1a). After 2 days in the hanging-drop culture, ESC-derived EBs were harvested, applied to a hanging-drop culture with new medium that contained $10^{-7} \mathrm{M}$ retinoic acid for 3 days, and subsequently cultured in a DME-based medium for an additional 
a

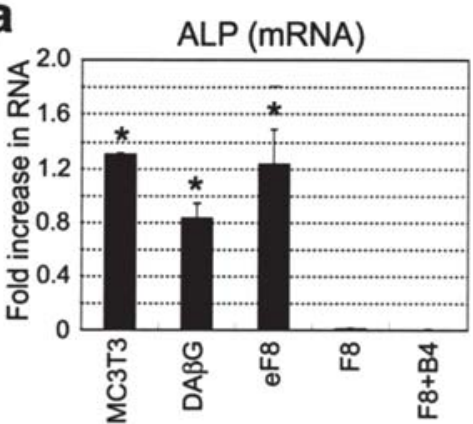

b

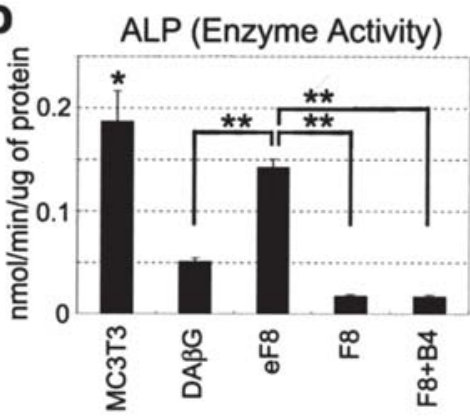

C

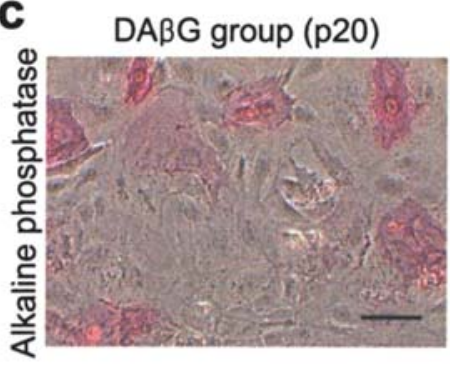

d

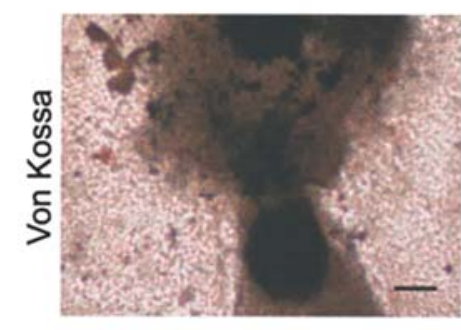

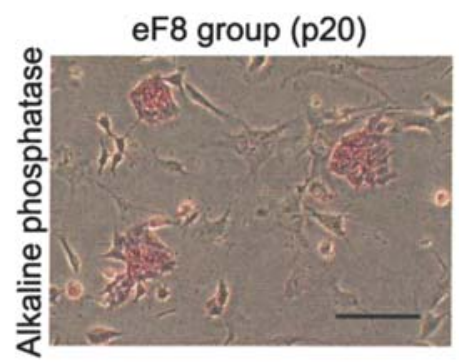

e

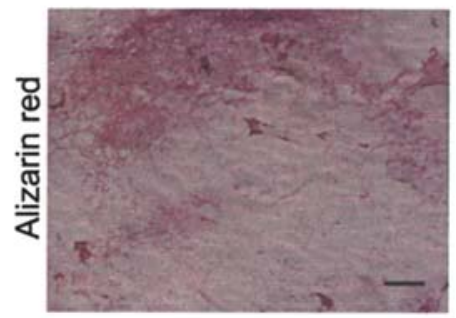

Figure 2. ALP expression and cytochemistry analysis of ESC-derived osteogenic cells. (a) ALP expression of ESC-derived osteogenic cells measured by realtime PCR analysis. ${ }^{*} \mathrm{p}<0.01$. (b) Enzyme activity of ALP in each group. The single asterisk indicates that the MC3T3 group is significantly higher than the other four groups. ${ }^{*} \mathrm{p}<0.01$. The double asterisk indicates that the eF8 group has a significantly higher value than those of the DAßG, F8, and F8+B4 groups. ${ }^{* *} \mathrm{p}<0.05$. (c) Representative images of ALP staining. Representative images of DAßG and eF8 groups were taken of cells that had been passaged 20 times after differentiation. Bars, $50 \mu \mathrm{m}$. Cytochemical analysis was performed using von Kossa staining (d) and Alizarin red staining (e). Bars, 1 mm.

a

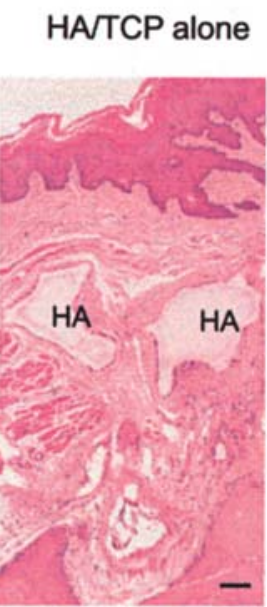

b
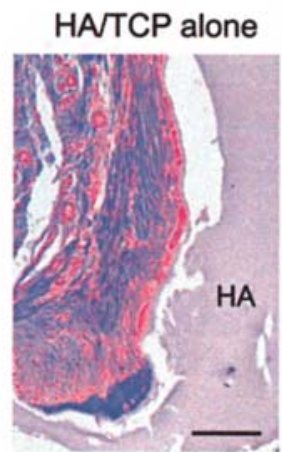

ESCs + HAVTCP

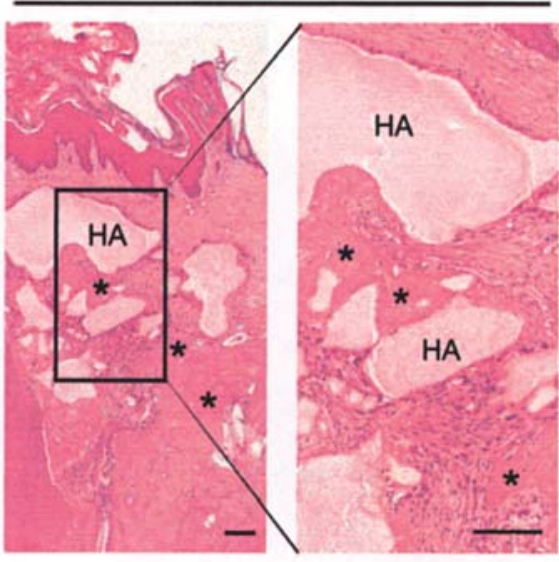

ESCs + HAVTCP

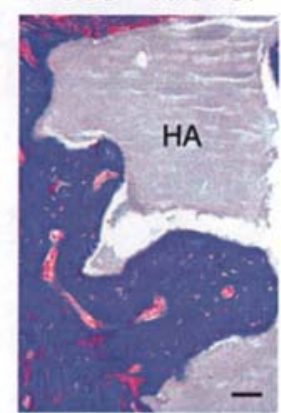

EBs

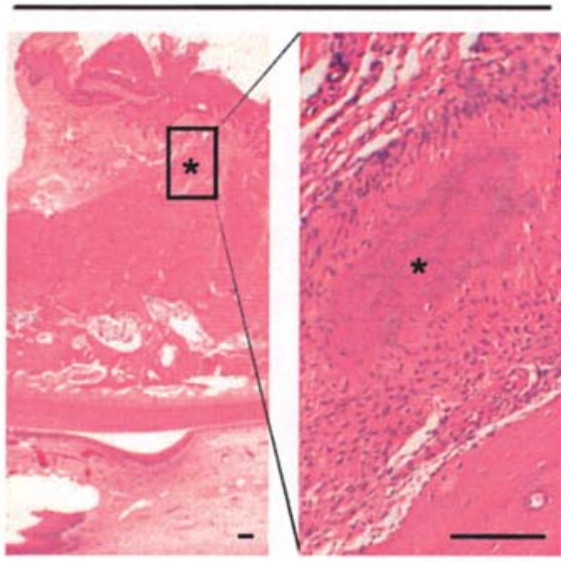

EBs

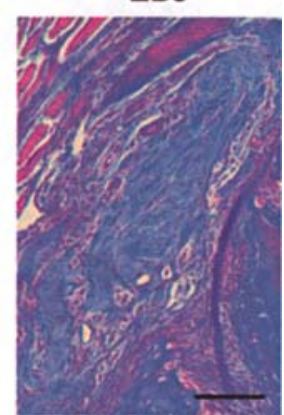

Figure 3. Transplantation of ESCs with HA/TCP or EBs into tooth sockets. (a) H\&E staining of HA/TCP alone, ESCs + HA/TCP, and EBs alone groups; HA stands for the HA/TCP regions. The asterisk shows the location of the newly formed bone structure. Each boxed area was enlarged in the left panel. (b) Mason Trichrome staining for collagen in each group. The blue area shows the deposition of collagen. Bars, $100 \mu \mathrm{m}$. 
2 days (Fig. 1b). Fully-grown EBs (day 7) were then harvested and cultured for an additional 21 days under different conditions as described in Materials and methods. Following differentiation toward osteogenic lineages, EB-derived cells were cultured for proliferation. Samples of ESCs, the feeder layer (STO), and the preosteoblast line (MC3T3-E1) taken at passage 20 were applied to real-time PCR analysis with various primers (Fig. 1c). Previous studies have reported that osteonectin, type I collagen, and osteopontin are early/intermediate markers for osteogenesis, while Cbfa-1, bone sialoprotein, and osteocalcin are intermediate/late markers (10). In this study, the results of real-time PCR analysis showed that the expression levels of osteonectin, type I collagen, and osteopontin, which are known as the early/ intermediate markers for osteogenic differentiation, were increased in the F8 group compared with the other groups. The transcription of intermediate/late markers, including Cbfa-1, bone sialoprotein, and osteocalcin, was upregulated in the eF8 group. These results demonstrated that FGF8 had an osteogenic effect on ESC-derived cells; however, the timedependent effect of FGF8 was not clear. The pre-osteoblast cells (MC3T3 group), which served as a positive control, showed enhanced expression of osteopontin, Cbfa-1, bone sialoprotein, and osteocalcin. However, other groups such as, ESC, DAßG, and F8+B4 did not exhibit significant expression of these markers. These results indicated that ESCs could differentiate into an osteogenic lineage, which was confirmed by real-time PCR analysis of specific bone lineage markers.

Characterization of in vitro differentiated osteogenic cells derived from mouse ESCs. In vitro osteogenesis of ESCs was characterized through the detection of ALP transcription/ activity, von Kossa staining, and Alizarin red staining. Both the DAßG and eF8 groups showed higher expressions of ALP than the ESCs, F8, and F8+B4 groups, which was determined by real-time PCR (Fig. 2a). Levels of ALP transcription in each of the MC3T3, DAßG, and eF8 groups were significantly higher than those of the F8 and F8+B4 groups. Moreover, ALP enzyme activity in the DAßG and eF8 groups was increased compared with that of the F8 and F8+B4 groups (Fig. 2b). More specifically, the ALP activity of the MC3T3 group was significantly higher than that of the other groups, while the ALP activity in the eF8 group was significantly elevated compared with that of the DAßG, F8, and F8+B4 groups. Cytochemistry analysis was used to confirm the existence of ALP activity in DAßG and eF8 groups (Fig. 2c). Likewise, our results showed that ESCs were strongly positive for ALP (data not shown). Further, the DAßG and eF8 groups (passage 20) contained ALP positive cells. Additional cytochemistry analyses using von Kossa (Fig. 2d) and Alizarin red stain (Fig. 2e) demonstrated calcium accumulation in the ESC-derived osteogenic cells. Together, these data indicated that osteogenic cells from ESCs could produce an accumulation of calcium and were positive for ALP activity.

In vivo osteogenic potential of ESCs and differentiated EBs in rat tooth sockets. To assess the in vivo bone-forming ability of ESCs and EBs, we transplanted HA/TCP (HA/TCP alone group), ESCs mixed with HA/TCP (ESCs + HA/TCP group), or an EBs pellet (EBs group) into rat tooth sockets without medication for immunosuppression. After 12 weeks, we sacrificed the recipient rats and retrieved the mandibular region for further analysis. We did not observe any formation of teratomas or tumors in the sacrificed rats. H\&E staining showed that there was no new osteoid tissue formation in the sham group (no transplantation in tooth sockets, data not shown) or the HA/TCP alone group (Fig. 3a), although in the latter group fibrosis and infiltration of giant cells was observed. In the ESCs + HA/TCP group, we observed distinct and widespread formation of immature bone tissue around the transplanted HA/TCP, but did not see evidence of fibrosis or infiltration of giant cells (Fig. 3a). Similarly to what was observed for the ESCs + HA/TCP group, we were able to identify the differentiation of immature bone tissue inside the sockets of the EBs group, and there was also no fibrosis or giant cells. To detect collagen deposition in the transplanted site, we used Mason Trichrome staining. In the ESCs + HA/TCP group as well as the EBs group, strong blue staining was present in the newly formed osteoid tissue, which implied collagen deposition (Fig. 3b). These data suggested that ESCs with HA/TCP and EBs were capable of differentiating into osteogenic tissue in tooth sockets.

\section{Discussion}

In this report, we demonstrated the osteogenic potential of ESCs in tooth sockets. The in vitro differentiation of mouse ESCs toward osteogenic lineages was achieved by hangingdrop culture and different culture conditions. Transplanted mouse ESCs mixed with HA/TCP or EBs alone in rat tooth sockets were able to differentiate and form osteoid structures.

Previously, in vitro differentiation of ESCs toward osteogenic tissues has been reported using low-density tissue culture, conditioned medium, addition of extracellular matrix, and three-dimensional scaffolding (11-14). Similarly, the present study demonstrated osteogenic differentiation from ESCs, which was confirmed by the increased expression of several osteoegenic markers such as osteonectin, type I collagen, osteopontin, Runx2, bone sialoprotein, and osteocalcin. Early/intermediate markers for osteogenesis (Runx2, bone sialoprotein, osteocalcin, and ALP) were highly transcribed in the F8 group, while the expression of intermediate/ late markers (Runx2, bone sialoprotein, and osteocalcin) was maintained in the eF8 group, thereby implying a stagedependent effect of FGF8 for osteogenesis from ESCs. FGF8 has been known as an important factor for limb and craniofacial skeletal structure, including bone $(15,16)$. Furthermore, mutations in the FGF signalling pathway have been implicated in several genetic diseases including Apert, Crouzon, Saethre-Chotzen, and Pfeiffer syndromes (17). BMP-4 is a well-known factor for differentiation toward mesodermal lineages from ESCs and a potent factor for ectopic osteogenesis in adult tissue (18-20). However, we did not find a significant enhancement of osteogenetic gene expression in the F8+B4 group, and our preliminary results showed that the other combinations of treatments (such as early, late, or early to late BMP4 alone or combined with FGF8) were unable to significantly alter genetic marker expression, ALP activity, or cytochemical staining (data not shown). In addition to genetic expression profiling, a cytochemistry assay was 
used in this study to characterize osteogenic differentiation. Alizarin red and von Kossa staining showed the calcium accumulation in ESC-derived cells, which was consistent with other studies (11).

ESC lines are known to be pluripotent and can give rise to multiple lineages $(21,22)$. Another characteristic of ESCs is their ability to induce teratoma formation in immune-deficient mice, which often consist of various tissues, including bone $(23,24)$. Likewise, adult stem cells such as bone marrow stem cells or mesenchymal stem cells have been reported to have differentiation potentials toward osteogenic tissues when transplanted with biomaterials or scaffolding materials such as alginate, HA, and hydrogel (25-27). In the present study, we transplanted ESCs mixed with HA/TCP into tooth sockets, and found that the ESCs could differentiate into osteogenic structures without teratoma formation. Moreover, the ESCderived EBs could form osteoid structures independent of biomaterials (HA/TCP), suggesting that the tooth socket environment might also be an osteogenic environment. While a similar effect has been observed in a canine alveolar socket model, the mechanism or source of possible signals for that environment has yet to be elucidated (9).

Taken together, we observed the differentiation potentials of ESC-derived EBs toward osteogenic tissues. ESC-derived osteogenic cells showed increased expression of various osteogenic markers, which was determined by both real-time PCR and cytochemical staining. When transplanted into a tooth socket environment, the mixtures of ESCs and HA/TCP particles or EBs alone successfully differentiated into osteogenic structures. These data suggest that ESCs may be an alternative source for craniofacial reconstruction.

\section{Acknowledgments}

This work was supported by a grant KRF-2004-042-E00127 from the Korea Research Foundation (to B.-M.M.).

\section{References}

1. Evans MJ and Kaufman MH: Establishment in culture of pluripotential cells from mouse embryos. Nature 292: 154-156, 1981 .

2. Wobus AM and Boheler KR: Embryonic stem cells: prospects for developmental biology and cell therapy. Physiol Rev 85: 635-678, 2005.

3. Chai Y and Slavkin HC: Prospects for tooth regeneration in the 21st century: a perspective. Microsc Res Tech 60: 469-479, 2003.

4. Gronthos S, Mankani M, Brahim J, Robey PG and Shi S: Postnatal human dental pulp stem cells (DPSCs) in vitro and in vivo. Proc Natl Acad Sci USA 97: 13625-13630, 2000.

5. Miura M, Gronthos S, Zhao M, Lu B, Fisher LW, Robey PG and Shi S: SHED: stem cells from human exfoliated deciduous teeth. Proc Natl Acad Sci USA 100: 5807-5812, 2003.

6. Seo BM, Miura M, Gronthos S, Bartold PM, Batouli S, Brahim J, Young M, Robey PG, Wang CY and Shi S: Investigation of multipotent postnatal stem cells from human periodontal ligament. Lancet 364: 149-155, 2004.

7. Ivanovski S, Gronthos S, Shi S and Bartold PM: Stem cells in the periodontal ligament. Oral Dis 12: 358-363, 2006.
8. Kawaguchi J, Mee PJ and Smith AG: Osteogenic and chondrogenic differentiation of embryonic stem cells in response to specific growth factors. Bone 36: 758-769, 2005.

9. De Kok IJ, Drapeau SJ, Young R and Cooper LF: Evaluation of mesenchymal stem cells following implantation in alveolar sockets: a canine safety study. Int J Oral Maxillofac Implants 20: 511-518, 2005.

10. Zur Nieden NI, Price FD, Davis LA, Everitt RE and Rancourt DE: Gene profiling on mixed embryonic stem cell populations reveals a biphasic role for beta-catenin in osteogenic differentiation. Mol Endocrinol 21: 674-685, 2007.

11. Chaudhry GR, Yao D, Smith A and Hussain A: Osteogenic cells derived from embryonic stem cells produced bone nodules in three-dimensional scaffolds. J Biomed Biotechnol 2004: 203-210, 2004

12. Woll NL, Heaney JD and Bronson SK: Osteogenic nodule formation from single embryonic stem cell-derived progenitors. Stem Cells Dev 15: 865-879, 2006.

13. Woll NL and Bronson SK: Analysis of embryonic stem cellderived osteogenic cultures. Methods Mol Biol 330: 149-159, 2006.

14. Woei Ng K, Speicher T, Dombrowski C, Helledie T, Haupt LM, Nurcombe $\mathrm{V}$ and Cool SM: Osteogenic differentiation of murine embryonic stem cells is mediated by fibroblast growth factor receptors. Stem Cells Dev 16: 305-318, 2007.

15. Albertson RC and Yelick PC: Fgf8 haploinsufficiency results in distinct craniofacial defects in adult zebrafish. Dev Biol 306: 505-515, 2007.

16. Moon AM and Capecchi MR: Fgf8 is required for outgrowth and patterning of the limbs. Nat Genet 26: 455-459, 2000.

17. Nie X, Luukko K and Kettunen P: FGF signalling in craniofacial development and developmental disorders. Oral Dis 12: 102-111, 2006.

18. Chen D, Zhao M and Mundy GR: Bone morphogenetic proteins. Growth Factors 22: 233-241, 2004.

19. Jane JA Jr, Dunford BA, Kron A, Pittman DD, Sasaki T, Li JZ, Li H, Alden TD, Dayoub H, Hankins GR, Kallmes DF and Helm GA: Ectopic osteogenesis using adenoviral bone morphogenetic protein (BMP)-4 and BMP-6 gene transfer. Mol Ther 6: 464-470, 2002.

20. Boyd NL, Dhara SK, Rekaya R, Godbey EA, Hasneen K, Rao RR, West FD III, Gerwe BA and Stice SL: BMP4 promotes formation of primitive vascular networks in human embryonic stem cellderived embryoid bodies. Exp Biol Med 232: 833-843, 2007.

21. Blum B and Benvenisty $\mathrm{N}$ : Clonal analysis of human embryonic stem cell differentiation into teratomas. Stem Cells 25: 1924-1930, 2007.

22. Martin GR: Isolation of a pluripotent cell line from early mouse embryos cultured in medium conditioned by teratocarcinoma stem cells. Proc Natl Acad Sci USA 78: 7634-7638, 1981.

23. Liu C, Chen Z, Chen Z, Zhang T and Lu Y: Multiple tumor types may originate from bone marrow-derived cells. Neoplasia 8: 716-724, 2006.

24. Bielby RC, Boccaccini AR, Polak JM and Buttery LD: In vitro differentiation and in vivo mineralization of osteogenic cells derived from human embryonic stem cells. Tissue Eng 10: 1518-1525, 2004.

25. Weber M, Steinert A, Jork A, Dimmler A, Thurmer F, Schutze N, Hendrich $\mathrm{C}$ and Zimmerman U: Formation of cartilage matrix proteins by BMP-transfected murine mesenchymal stem cells encapsulated in a novel class of alginates. Biomaterials 23: 2003-2013, 2002.

26. Boo JS, Yamada Y, Okazaki Y, Hibino Y, Okada K, Hata K, Yoshikawa T, Sugiura Y and Ueda M: Tissue-engineered bone using mesenchymal stem cells and a biodegradable scaffold. J Craniofac Surg 13: 231-233, 2002.

27. Endres M, Hutmacher DW, Salgado AJ, Kaps C, Ringe J, Reis RL, Sittinger M, Brandwood A and Schantz JT: Osteogenic induction of human bone marrow-derived mesenchymal progenitor cells in novel synthetic polymer-hydrogel matrices. Tissue Eng 9: 689-702, 2003. 